\title{
Dermopathy associated with cetuximab and panitumumab: investigation of the usefulness of moisturizers in its management
}

This article was published in the following Dove Press journal:

Clinical, Cosmetic and Investigational Dermatology

II September 2017

Number of times this article has been viewed

\author{
Shoichi Watanabe' \\ Motoki Nakamura' \\ Hiroki Takahashi ${ }^{2}$ \\ Masayasu Hara ${ }^{2}$ \\ Kei ljichi ${ }^{3}$ \\ Daisuke Kawakita ${ }^{3}$ \\ Akimichi Morita' \\ 'Department of Geriatric and \\ Environmental Dermatology, \\ ${ }^{2}$ Department of Gastroenterological \\ Surgery, ${ }^{3}$ Department of Neuro- \\ otolaryngology, Nagoya City \\ University Graduate School of \\ Medical Science, Nagoya, Japan
}

\begin{abstract}
Aims: Limited data regarding the objective evaluation of skin exsiccation caused by epidermal growth factor receptor (EGFR) inhibitors exist. Objective indices were applied to evaluate the usefulness of a moisturizer against skin exsiccation associated with the use of EGFR inhibitors in cancer patients.
\end{abstract}

Patients and methods: Patients with either colorectal or head and neck cancer treated with either cetuximab or panitumumab were randomly assigned 1:2 to the prophylactic-treatment arm, where participants received prophylactical moisturizer treatment (heparinoid preparation, Hirudoid $^{\circledR}$ ), or to the symptomatic-treatment arm, where moisturizer was applied after the onset of cutaneous symptoms. Patients were observed for 6 weeks after the start of the administration of EGFR inhibitor.

Results: A total of 15 patients were included and assessed: 6 in the prophylactic treatment group and 9 in the symptomatic treatment group. We evaluated the effect of EGFR inhibitors on the physiologic function of the skin. Administration of EGFR inhibitors resulted in a continued reduction in the moisture content of the horny layer and a decrease in cutaneous sebum levels. Skin observation revealed frequent development of an acneiform rash and significantly higher exsiccation and exfoliation scores. Administration of EGFR inhibitor was associated with a decrease in the horny layer moisture content and lower cutaneous sebum levels in the symptomatic treatment group. In contrast, levels of both the indicators were increased in the prophylactic treatment group. Moreover, the EGFR inhibitor-associated increase in exsiccation and exfoliation scores were minimized in the prophylactic treatment group relative to the symptomatic treatment group.

Conclusion: Application of a moisturizer is effective against dry skin induced by the administration of an EGFR inhibitor.

Keywords: EGFR inhibitor, moisturizer, colorectal cancer, head and neck cancer, prophylactical moisturizer treatment, heparinoid preparation, skin exsiccation

\section{Introduction}

Epidermal growth factor receptor (EGFR) inhibitors, which are molecular target agents used to treat cancer, often induce dermopathies such as an acneiform rash, dry skin, and pruritus. ${ }^{1-3}$ Among the dermopathies triggered by the administration of an EGFR inhibitor, limited data are available regarding objective evaluations of skin exsiccation. ${ }^{4}$ This small clinical study aimed to objectively evaluate the effects of the EGFR inhibitors cetuximab and panitumumab on the physiologic functions of the skin. By applying objective indices, we examined the usefulness of a moisturizer for the management of skin exsiccation induced by the administration of an EGFR inhibitor.
Correspondence: Shoichi Watanabe Department of Geriatric and Environmental Dermatology, Nagoya City University Graduate School of Medical Sciences, No. 3 I-Kawasumi Mizuho-cho, Mizuho-ku, Nagoya 467-860I, Japan Tel +8I 52853826 I

Email wshoichi@gmail.com 


\section{Patients and methods}

In collaboration with Nagoya City University and the nonprofit organization JASMIN, this open-label, randomized comparison study was conducted after review and approval by the institutional review board of Nagoya City University. The study is registered in the Clinical Trials Registry (UMIN-CTR) of the University Hospital Medical Information Network (UMIN) with the trial ID UMIN000019896.

Patients with colorectal or head and neck cancer aged $\geq 20$ years who were administered either cetuximab or panitumumab were included in the study. Patients were excluded from the study if they had a history of EGFR inhibitor treatment, suffered from complications or a history of dermopathy in extensive regions, had hemopathies with hemorrhagic symptoms, had used topical agents with retinoid-like effects within 2 weeks prior to starting the trial, and/or were diagnosed with drug-associated skin eruptions. Before the study began, written informed consent was obtained from all the participants.

Patients were randomly assigned 1:2 to the prophylactic treatment arm, where they were administered a moisturizer prophylactically, and the symptomatic treatment arm, where they were instructed to apply a moisturizer following symptom onset. Patients were observed for 6 weeks after the start of the administration of the EGFR inhibitor. Observation target regions were face, thorax, and lateral upper arm (left or right). A heparinoid preparation (Hirudoid $^{\circledR}$; Maruho, Osaka, Japan) was used as the moisturizer (with humectant function) and supplied to the subjects. The heparinoid preparation, which is often used in Japan for dermatologic care, is reported to be superior to urea preparations or petrolatum with respect to dermal outcomes..$^{5-7}$ The symptomatic treatment group was supplied with the topical moisturizer when exsiccation of grade $\geq 2$ was noted according to the Common Terminology Criteria for Adverse Events (CTCAE) v4.0. The selection of the drug formulation (ointment, cream, or lotion) was at the discretion of each dermatologist. An optimum dose of the moisturizer was applied at least twice daily to the target region. Based on a previous report concerning the volume of the ointment, the optimum dose was decided and the amount of 1 finger-tip unit was applied to the target site, so that it was equivalent to two palms. ${ }^{8}$ In principle, the use of other external agents to the target region was prohibited. Patients also received two 50-mg minocycline hydrochloride tablets, twice daily, as a prophylactic treatment against an acneiform rash. Patients were exempt from the oral minocycline treatment if it was not considered suitable.
Prior to the start of administration of EGFR inhibitor and 1-6 weeks after initiation of the treatment, subjective skin symptoms (pruritus) and objective findings (exsiccation and exfoliation) were evaluated every week during the observation period. The horny layer moisture content and level of cutaneous sebum were also measured. A dermatologist observed photographs of the target region and evaluated skin symptoms on a 5 -scale score of 0 (none) to 4 points (severe) with a visual scale. Development of acneiform rash, erythema, and wheals was also evaluated. The MPA 5 (Courage-Khazaka, Cologne, Germany) was used to measure the horny layer moisture content and sebum level. MPA 5 is a multi-skin measuring instrument which contains a corneometer for measuring horny layer moisture content and a sebumeter for measuring sebum level. The corneometer and the sebumeter use a dedicated probe for measuring. The corneometer instantaneously measures the moisture content of the stratum corneum by measuring the electrostatic capacitance generated on the skin. The measured value is displayed as a relative value of capacitance (unit: $\mathrm{AU})$. The sebumeter measures the amount of sebum secretion by attaching a tape to the skin surface. After selecting one measured region of $\sim 3 \times 3 \mathrm{~cm}$ in each target region, the cutaneous sebum level was measured. Each region of skin was then washed with a mild detergent (acidulent face soap, 2 $\mathrm{e}^{\circledR}$; Shiseido, Tokyo, Japan) or lukewarm water and acclimated with a 20-minute rest before measuring the horny layer moisture content. Within each target region, five measurements of the horny layer moisture content and four measurements of the sebum level were obtained at each distinct spot. The mean of the multiple measurements was adopted.

The primary endpoints of the study included the measurements of the horny layer moisture content and sebum level at the onset of dermopathy, the horny layer moisture content in the stratum corneum, and dermatologic findings (exsiccation and exfoliation) as indices of the moisturizer effectiveness. Statistical analysis was performed with the one-sample $t$-test for the assessment of the horny moisture content and sebum level before and after the administration of EGFR inhibitor and the two-sample $t$-test for between-arm comparisons. The Wilcoxon signed-rank test was used to compare the dermatologic scores of exsiccation and exfoliation. The significance level was set at a hazard ratio of $<5 \%(p<0.05$, two-tailed test $)$.

\section{Results}

\section{Patient characteristics}

A total of 22 patients were enrolled between January 2013 and December 2015; 7 withdrew either due to the termination of EGFR inhibitor administration or for other reasons. 
Patients were randomized to either the prophylactic treatment arm $(n=8)$ or the symptomatic treatment arm $(n=14)$. In the prophylactic treatment arm, two patients dropped out; one patient had not used moisturizer prophylactically and another had high fever. In the symptomatic treatment arm, five patients were excluded because of tumor progression, pneumothorax, and anaphylaxis to EGFR inhibitor. Thus, 15 patients were included in the statistical analysis -6 patients in the prophylactic treatment arm (mean age 64 years, 5 men) and 9 (mean age 71 years, 7 men) in the symptomatic treatment arm; by primary cancer type, 7 colorectal cancer patients and 8 head and neck cancer patients; by EGFR inhibitor treatment, 12 (7 in the symptomatic treatment arm) received cetuximab and 3 ( 2 in the symptomatic treatment arm) received panitumumab. Prophylactic administration of minocycline was performed in all the cases. No patient in the symptomatic treatment arm received any moisturizers over the course of the study.
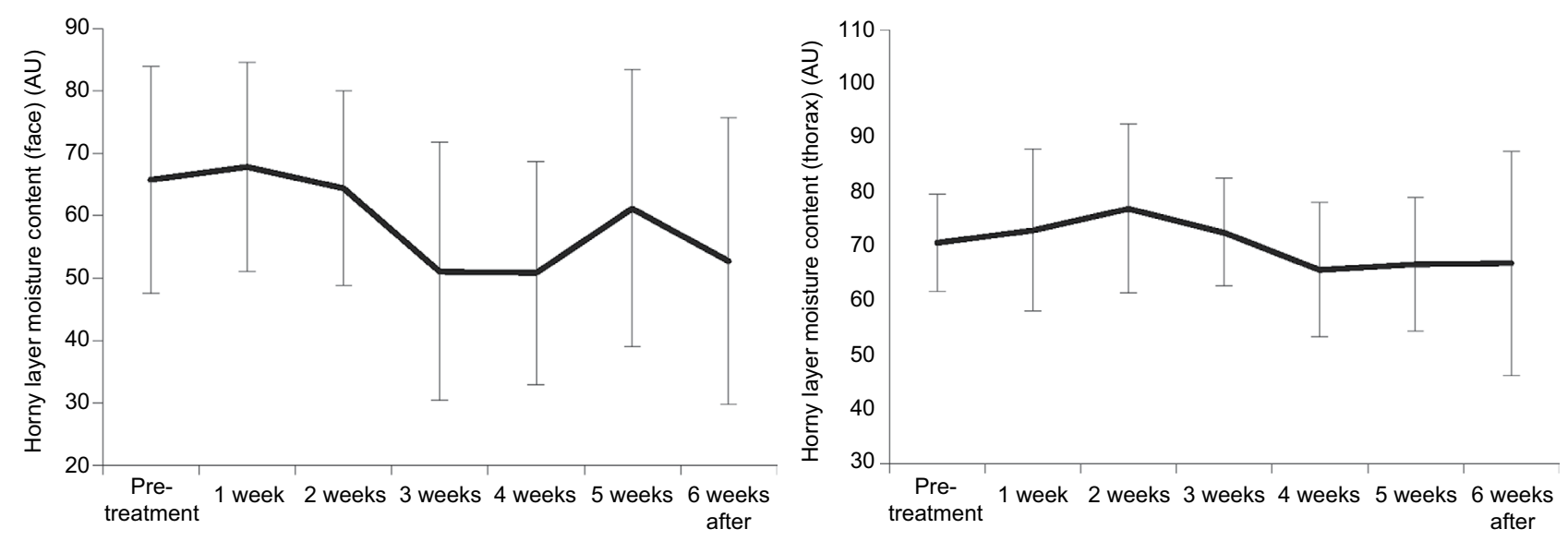

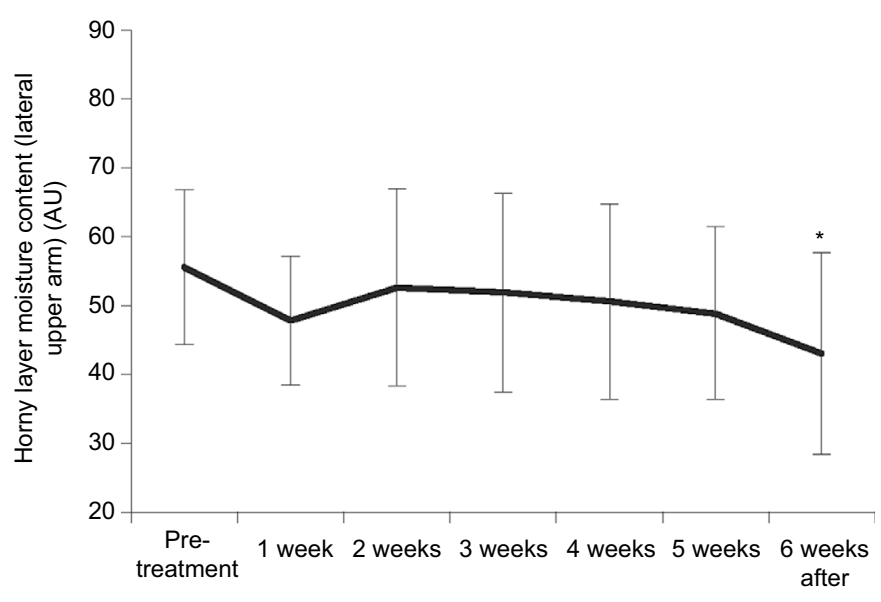

Figure I Cutaneous effects of administration of EGFR inhibitor: variation in the horny layer moisture content (symptomatic treatment arm). Notes: ${ }^{*} p<0.05$ (vs pretreatment baseline, paired $t$-test). Error bars indicate SD.

Abbreviation: EGFR, epidermal growth factor receptor. 
noted in facial sebum levels, with the mean value rising slightly from the pre-administration level of 75.06 to $76.19 \mu \mathrm{g} / \mathrm{cm}^{2}$ at 6 weeks ( $p=0.952$ ), sebum levels decreased from 17.22 to $7.47 \mu \mathrm{g} / \mathrm{cm}^{2}(p=0.160)$ on the thorax and from 6.22 to $0.56 \mu \mathrm{g} /$ $\mathrm{cm}^{2}(p=0.133)$ on the lateral upper arm, respectively (Figure 2 ).

\section{Observed changes in skin conditions after the administration of EGFR inhibitor}

The development of an acneiform rash observed following the treatment with an EGFR inhibitor was most frequent at 2 weeks posttreatment; this symptom also persisted thereafter (Figure 3). The development of an acneiform rash on the face at this time point was observed in up to $55.6 \%$ of patients (compared with $0.0 \%$ before treatment initiation). At 6 weeks, an acneiform rash on the face was observed in $22.2 \%$ of patients. On the thorax, although the incidence was lower than that on the face, an acneiform rash was observed in $22.2 \%$ of patients at 2 weeks posttreatment (compared with a rate of $0.0 \%$ before the initiation of the treatment); $11.1 \%$ of patients had an acneiform rash at 6 weeks. No patient in the study experienced an acneiform rash on the lateral upper arm.

The exsiccation scores in all the regions were significantly increased 6 weeks after beginning the treatment, from 0.00 to $1.33(p=0.010)$ on the face, from 0.22 to $1.33(p=0.008)$ on the thorax, and from 0.22 to $1.44(p=0.008)$ on the lateral upper arm (Figure 4). The exfoliation scores were also significantly higher; from 0.00 before the initiation of the treatment to $1.00(p=0.014)$ on the face 6 weeks after the first dose was administered, from 0.00 to $0.67(p=0.034)$ on the thorax, and from 0.00 to $0.78(p=0.020)$ on the lateral upper arm (data not shown).

\section{Usefulness of moisturization}

Figure 5 shows the variation in the layer moisture content before and after the administration of the EGFR inhibitor in the prophylactic treatment arm and in the symptomatic treat-
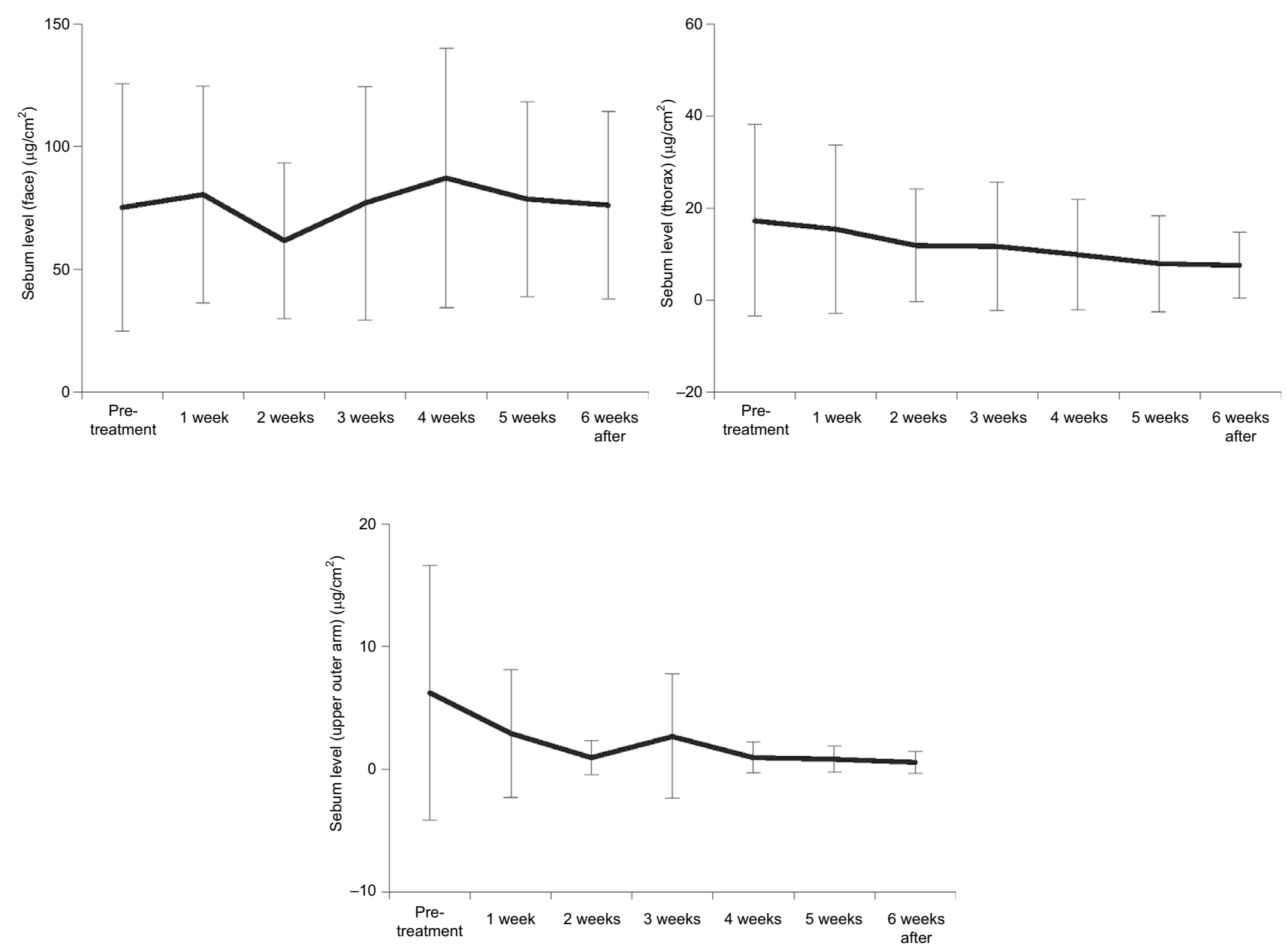

Figure 2 Cutaneous effects of administration of EGFR inhibitor: variation in the sebum level (symptomatic treatment arm). Notes: Error bars indicate SD.

Abbreviation: EGFR, epidermal growth factor receptor. 


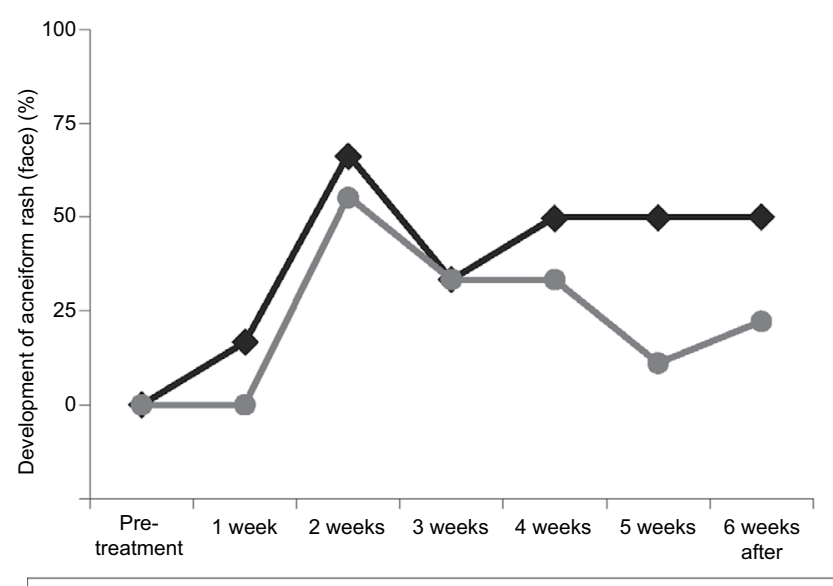

Group A : prophylactic treatment arm $\rightarrow$ Group B : symptomatic treatment arm

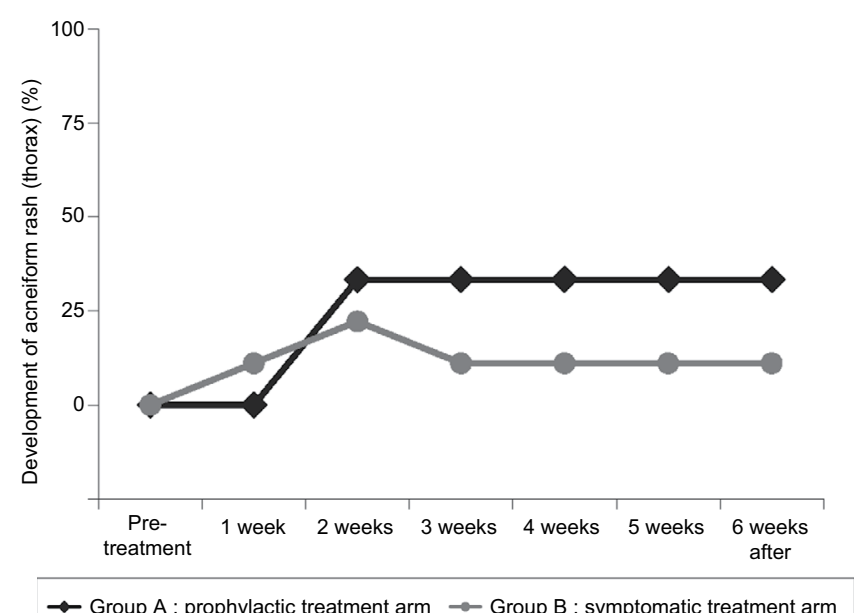

$\multimap$ Group A : prophylactic treatment arm $\rightarrow$ Group B : symptomatic treatment arm

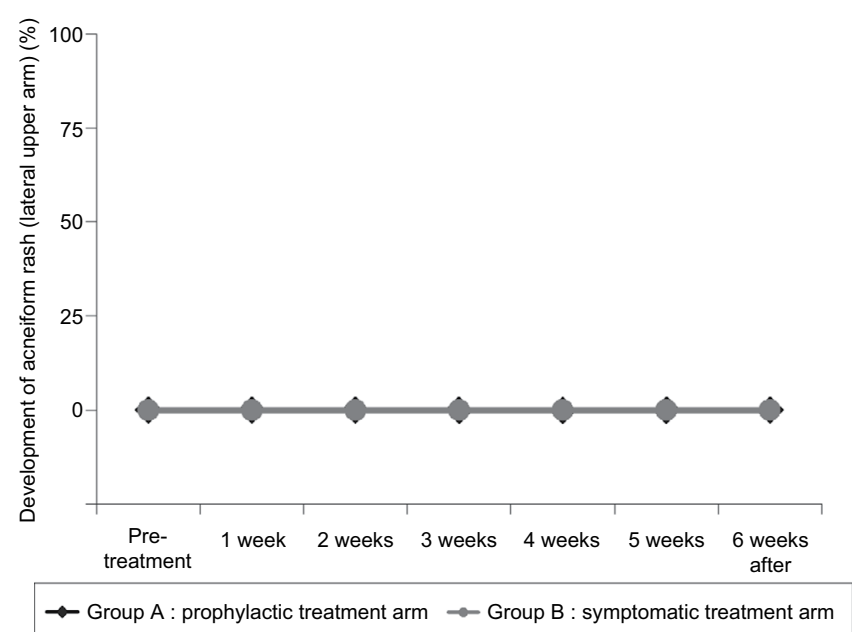

Figure 3 Development of EGFR inhibitor-induced acneiform rash: positive cases. Abbreviation: EGFR, epidermal growth factor receptor.

ment arm. While the horny layer moisture content dropped in all the target regions in the symptomatic treatment group (from -3.76 to $-13.00 \mathrm{AU}$ ), the horny layer moisture content increased on the thorax $(+7.64 \mathrm{AU}, p=0.158)$ and on the lateral upper arm $(+0.07 \mathrm{AU}, p=0.986)$ in the prophylactic treatment group.

A similar difference was observed in the variation of the cutaneous sebum level. In the symptomatic treatment group, sebum levels on the thorax and the lateral upper arm decreased (between -5.67 and $-9.75 \mu \mathrm{g} / \mathrm{cm}^{2}$ ). In contrast, sebum levels in all the target regions increased in the prophylactic treatment group (face: $+10.92 \mu \mathrm{g} / \mathrm{cm}^{2}, p=0.370$, thorax: $+2.21 \mu \mathrm{g} / \mathrm{cm}^{2}, p=0.758$, lateral upper arm: $+0.38 \mu \mathrm{g} /$ $\mathrm{cm}^{2}, p=0.268$; Figure 6).
Skin observation revealed no significant difference in the number of acneiform rashes between the two groups, suggesting that external application of a moisturizer does not affect the development of acneiform rash (Figure 3).

In the symptomatic treatment group, exsiccation scores on the face, thorax, and lateral upper arm increased by 1.33 , 1.11 , and 1.22 from the baseline values before the administration of the treatment, respectively. The score increments in the prophylactic treatment group were limited to 1.50 , 0.66 , and 0.50 in the target regions, respectively (Figure 4). The exfoliation scores in the symptomatic treatment group increased by $1.00,0.67$, and 0.78 in the target regions and by $1.17,0.50$, and 0.50 in the prophylactic treatment group, respectively (data not shown). 


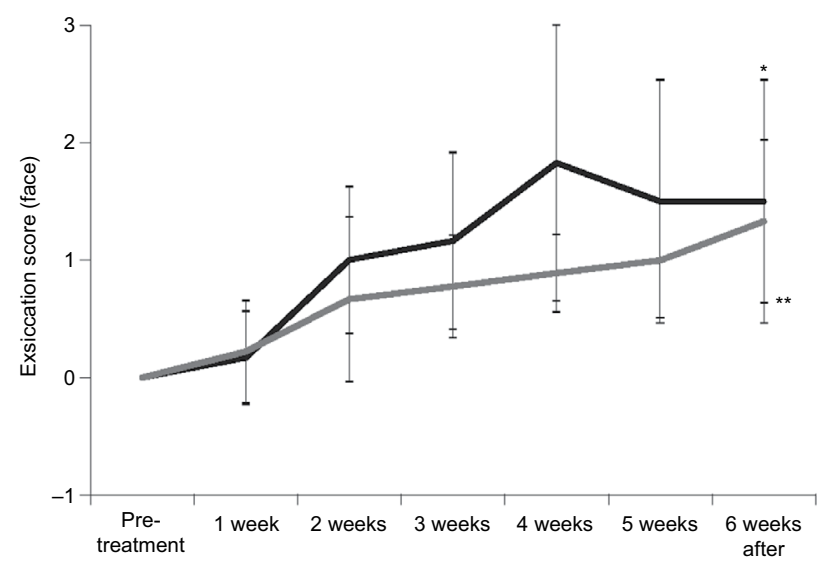

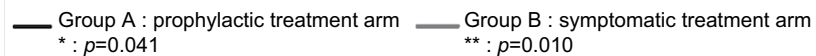

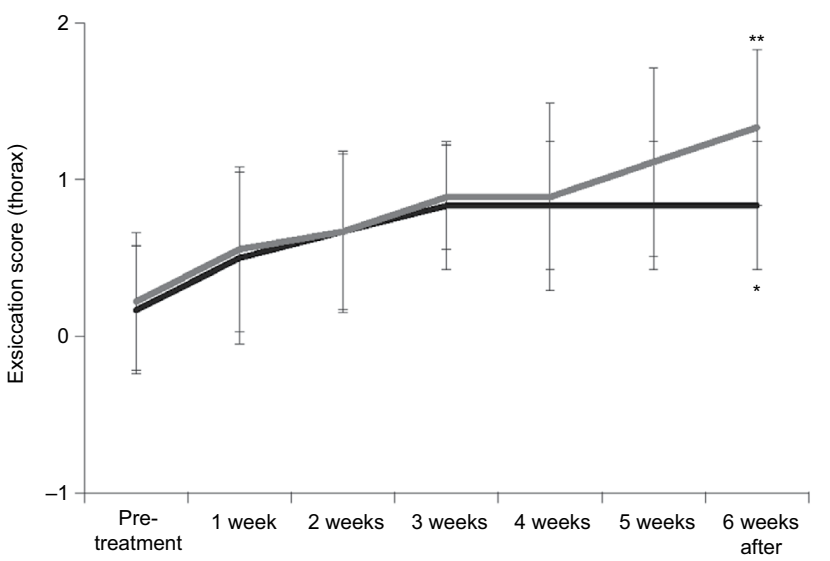

Group A : prophylactic treatment arm __ Group B : symptomatic treatment arm ${ }^{*}: p=0.046$

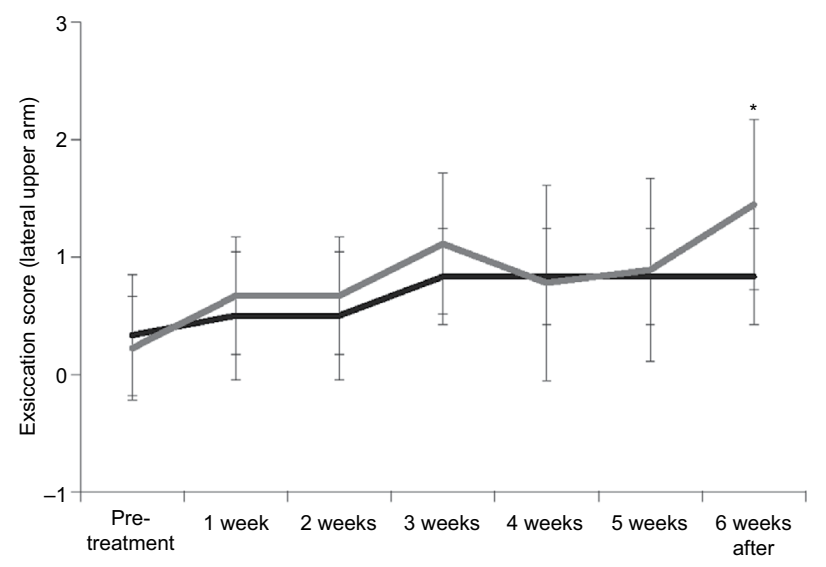

_ Group A : prophylactic treatment arm __ Group B : symptomatic treatment arm

Figure 4 EGFR inhibitor-induced variation in the dermatologic observation for exsiccation ( 0 - none, I - minimal, 2 - mild, 3 - moderate, 4 - severe). Notes: Error bars indicate SD. Wilcoxon signed-rank test, $p<0.05$.

Abbreviation: EGFR, epidermal growth factor receptor.

During the observation period, no adverse events attributable to topical moisturizer application were observed.

\section{Discussion}

Administration of EGFR inhibitors is frequently associated with dermopathy, with a particularly high incidence of acneiform rash. Despite concomitant prophylactic treatment against an acneiform rash with oral minocycline hydrochloride, we observed numerous cases with an acneiform rash, with an incidence as high as $55.6 \%$ on the face and $22.2 \%$ on the thorax, peaking at 2 weeks after beginning EGFR inhibitor administration.

The main objective of this study was to collect the fundamental information on dermopathies, particularly skin exsiccation, associated with the administration of EGFR. Therefore, the primary endpoints included were of an objective nature, such as the horny layer moisture content and the cutaneous sebum level over time, as well as the skin conditions evaluated by dermatologists. Scores for cutaneous symptoms such as exsiccation and exfoliation increased over time following the administration of the EGFR inhibitor. When levels before the administration of the EGFR inhibitor were compared with those 6 weeks after the initial dose was administered, the score increase was statistically significant in all the target regions. Moreover, an EGFR inhibitor-induced reduction in the horny layer moisture content and the cutaneous sebum level was also observed, with the development of exsiccation demonstrated by instrumental measurement. 

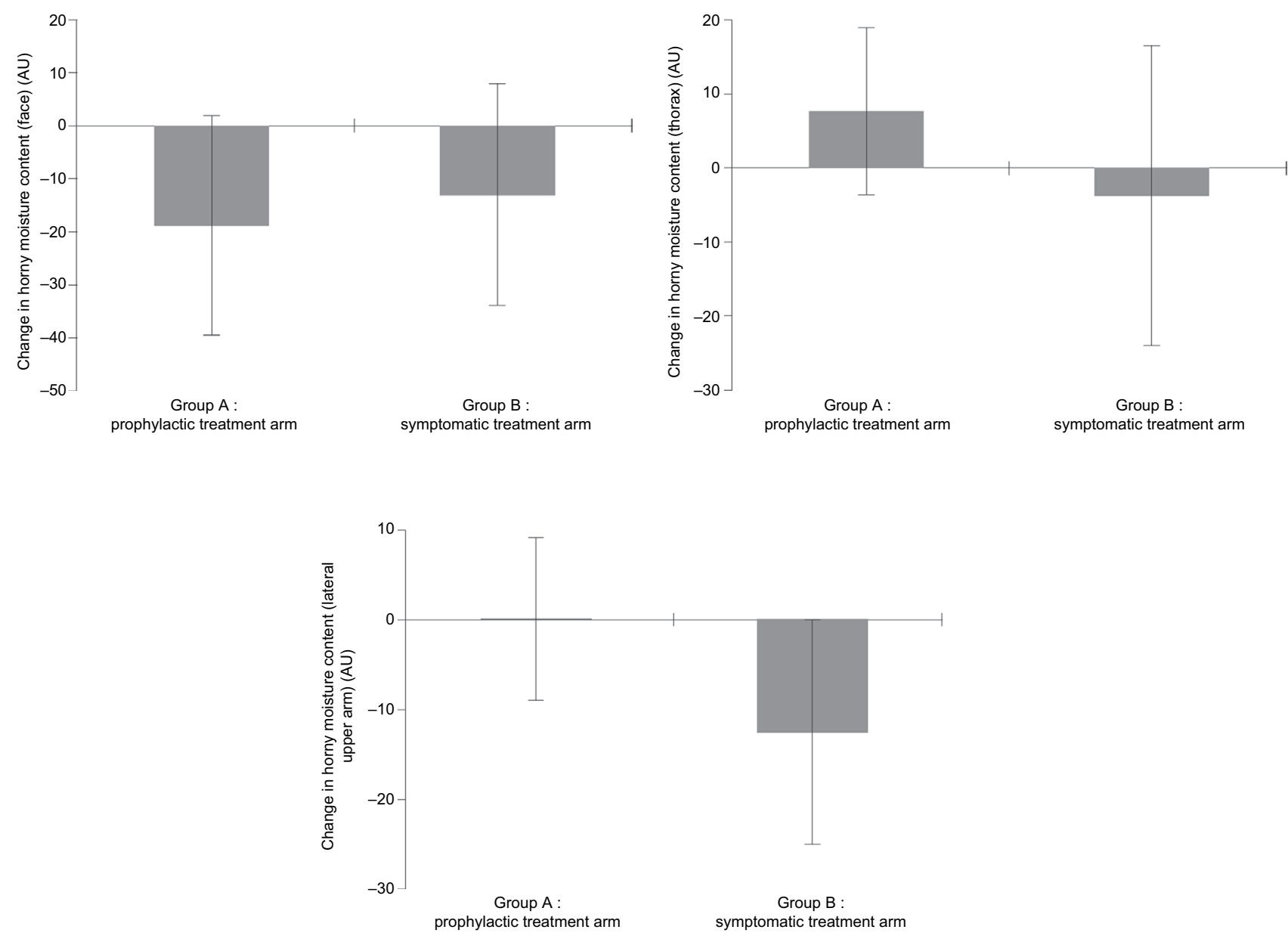

Figure 5 Usefulness of a moisturizer: comparison in the EGFR inhibitor-induced variation in the horny layer moisture content (0-6 weeks). Notes: Error bars indicate SD.

Abbreviation: EGFR, epidermal growth factor receptor.

The present study not only provides evidence of exsiccation expressed in numerical values based on instrumental measurement but also provides an objective evaluation by dermatologists who do not play an integral role in cancer treatment with EGFR inhibitors.

This clinical study also examined the usefulness of moisturizer against exsiccation, adopting the horny moisture content as an objective index. While the horny layer moisture content was reduced in all target regions in the group that did not use topical moisturizers, an increase in horny layer moisture content was observed in 2 of the 3 target regions in the group that administered the moisturizer. Furthermore, the dermatologic findings also support the usefulness of a moisturizer against EGFR inhibitor-induced exsiccation, with a lower incidence of dermopathy observed in the treatment group than in the control group.
A limitation of the present study is the small number of participants with two different primary cancer types and the use of two different EGFR inhibitors, which likely contributed to the overall large statistical variability in this study. Furthermore, the inclusion of some terminal cancer patients presented some challenges with regard to protocol compliance.

Despite these limitations, we believe our efforts to collect fundamental information on EGFR inhibitor-induced dermopathy, particularly exsiccation, were successful. In the context that EGFR inhibitor-induced dermopathy is now more widely recognized and prophylactic treatment with moisturizers has become more prevalent, ${ }^{9,10}$ the findings of this study documenting the natural history of the skin conditions during the administration of an EGFR inhibitor are considered meaningful. 

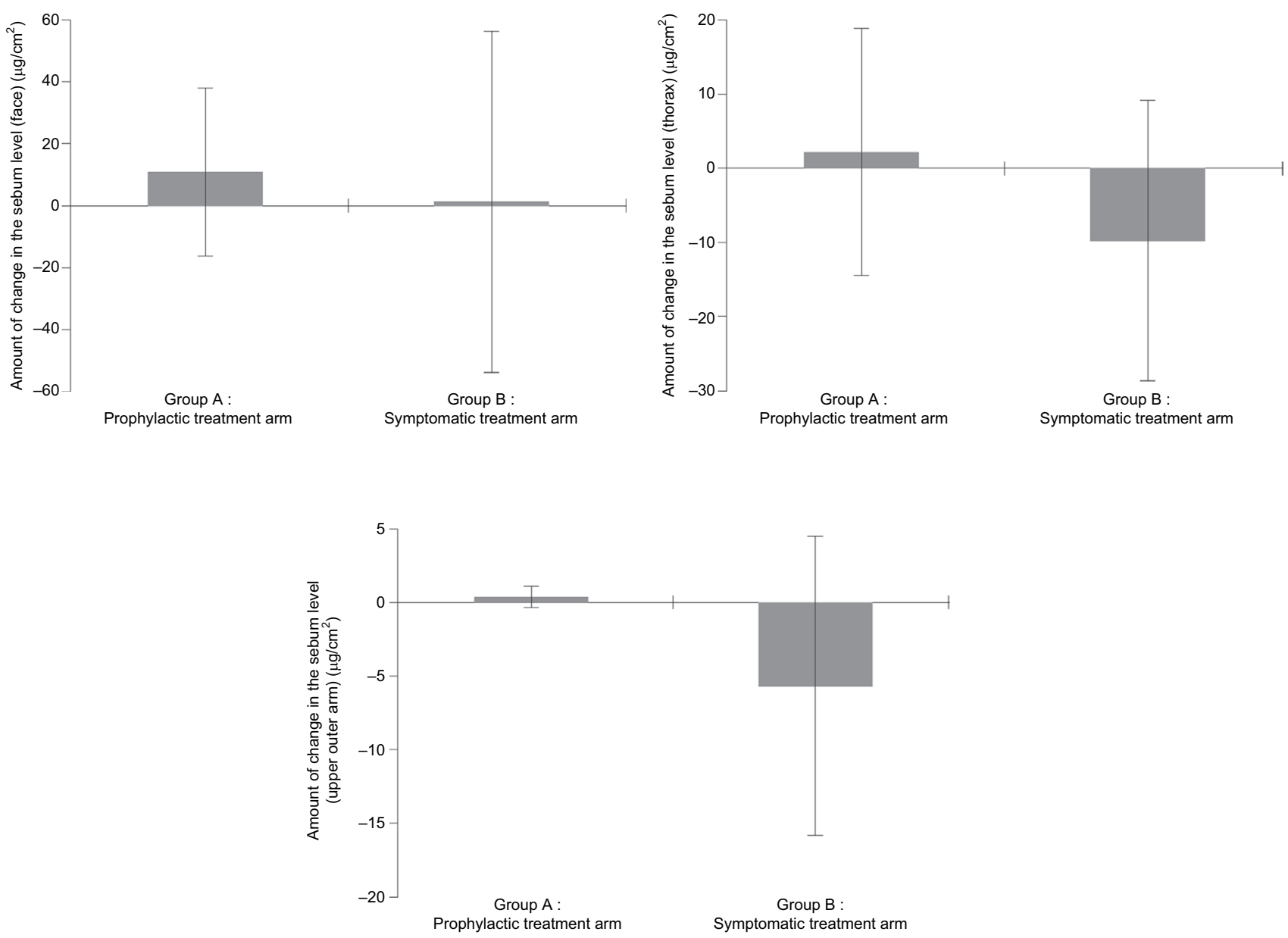

Figure 6 Usefulness of a moisturizer: comparison of the EGFR inhibitor-induced variation in the cutaneous sebum level (0-6 weeks).

Notes: Error bars indicate SD.

Abbreviation: EGFR, epidermal growth factor receptor.

\section{Acknowledgment}

The authors thank the patients who participated in the study.

\section{Disclosure}

The authors report no conflicts of interest in this work.

\section{References}

1. Jonker DJ, O'Callaghan CJ, Karapetis CS, et al. Cetuximab for the treatment of colorectal cancer. N Engl J Med. 2007;357(20):2040-2048.

2. Wacker B, Nagrani T, Weinberg J, Witt K, Clark G, Cagnoni PJ. Correlation between development of rash and efficacy in patients treated with the epidermal growth factor receptor tyrosine kinase inhibitor erlotinib in two large phase III studies. Clin Cancer Res. 2007;13(13):3913-3921.

3. Giovannini M, Gregorc V, Belli C, et al. Clinical significance of skin toxicity due to EGFR-Targeted therapies. J Oncol. 2009;2009:849051:1-8.

4. Nakahara T, Moroi Y, Takayama K, et al. Change in sebum levels and the development of acneiform rash in patients with non-small cell lung cancer after treatment with EGFR inhibitors. Onco Targets Ther. 2015;28(8):259-263.
5. Kawashima M, Ishizaki C. [Evaluation of the efficacy of medical moisturizers on artificially dried skin and atopic dry skin on the basis of dermato-physiological parameters]. Jpn J Dermatol. 2007;117(3): 275-284. Japanese.

6. Kawashima M, Numano K, Ishizaki C. [Usefulness of moisturizers for dermato-physiological dysfunction in patients with atopic dermatitis]. Jpn J Dermatol. 2007;117(6):969-977. Japanese.

7. Kawashima M, Numano K, Ishizaki C. [Usefulness of moisturizers for artificially dried skin in healthy subjects]. J Clin Ther Med. 2007;61(7):563-568. Japanese.

8. Long CC, Finlay AY. The finger-tip unit--a new practical measure. Clin Exp Dermatol. 1991;16(6):444-447.

9. Lacouture ME, Mitchell EP, Piperdi B, et al. Skin toxicity evaluation protocol with panitumumab (STEPP), a phase II, open-label, randomized trial evaluating the impact of a pre-Emptive Skin treatment regimen on skin toxicities and quality of life in patients with metastatic colorectal cancer. J Clin Oncol. 2010;28(8):1351-1357.

10. Kobayashi Y, Komatsu Y, Yuki S, et al. Randomized controlled trial on the skin toxicity of panitumumab in Japanese patients with metastatic colorectal cancer: HGCSG1001 study; J-STEPP.Future Oncol. 2015;11(4): $617-627$. 


\section{Publish your work in this journal}

Clinical, Cosmetic and Investigational Dermatology is an international, peer-reviewed, open access, online journal that focuses on the latest clinical and experimental research in all aspects of skin disease and cosmetic interventions. This journal is included on PubMed. The manuscript management system is completely online and includes a very quick and fair peer-review system, which is all easy to use. Visit http://www.dovepress.com/testimonials.php to read real quotes from published authors

Submit your manuscript here: https://www.dovepress.com/clinical-cosmetic-and-investigational-dermatology-journal 